\title{
Magnetic field measurements in Rb vapor by splitting Hanle resonances under the presence of a perpendicular scanning magnetic field
}

\author{
Raghwinder Singh Grewal ${ }^{\mathrm{a}, \mathrm{b}}$ and Murari Pattabiraman \\ Department of Physics, Indian Institute of Technology Madras, Chennai 600036, India
}

Received 6 April 2016 / Received in final form 14 June 2016

Published online 25 October 2016

(C) The Author(s) 2016. This article is published with open access at Springerlink.com

\begin{abstract}
We experimentally and computationally study the effect of an additional transverse magnetic field (TMF) on the Hanle resonance for a $F_{g}=2 \rightarrow F_{e}=3$ transition of ${ }^{87} \mathrm{Rb} D_{2}$ line for magnetic field scans perpendicular to the propagation direction of the optical field. It is shown that with a $\pi$-polarized light, no resonance signal is observed in absence of the TMF. When the TMF is applied, two peaks are observed on either side of zero scanning magnetic field in the transmission spectrum. The separation between the two peaks is linearly proportional to magnitude of the TMF, which can be used for magnetometry. We applied this technique to measure magnetic field from 0.1 to $0.5 \mathrm{G}$ with a pure Rb vapor cell and from 10 to $30 \mathrm{mG}$ with a $\mathrm{Rb}$ cell containing buffer gas. These observations are attributed to the population redistribution among the ground state sublevels due to the TMF.
\end{abstract}

\section{Introduction}

Light-induced atomic orientation and alignment have been extensively explored to create atomic magnetometers based on nonlinear magneto optical rotation [1-4], optical pumping [5-7], and coherent population trapping [8-10]. These magnetometers operate by detecting the precession of the atomic orientation/alignment in the presence of a magnetic field via light transmission measurements [11]. The ground state Hanle effect has also been used to measure the magnetic field using optical pumping and radio frequency field setup $[6,7]$.

In the Hanle configuration, atomic-coherence induced phenomenon like electromagnetically induced transparency (EIT) and absorption (EIA) resonances are measured by scanning a magnetic field, either parallel to the direction of propagation of the optical field $[12,13]$ or perpendicular to the direction of propagation of the optical field [14-16].

In this paper, we report a new technique for measuring magnetic field using Hanle resonances with both pure and buffer gas cells of Rb. A single linearly polarized laser beam, called pump-probe beam, is used to polarize the atoms and to probe the absorption. When the scanning magnetic field is perpendicular to the propagation direction of the optical field, with a $\pi$ polarized light, no Hanle resonance signal is observed, since there is no change of population among ground state sublevels as a

\footnotetext{
a e-mail: raghugrewal.singh@gmail.com

b Current address: Institute of Physics, Jagiellonian University, Krakow, Poland
}

function of the scanning magnetic field. As a result, no coherence develops among the ground state sublevels. In the above configuration, when an additional magnetic field is applied perpendicular to the scanning magnetic field, it redistributes the population and creates the coherence among the ground state sublevels. As a result, two dips are observed in absorption spectrum on either side of the zero scanning magnetic field. The separation between the two dips in the Hanle absorption spectrum is proportional to the magnitude of the additional transverse magnetic field (TMF), and can be used to measure the magnitude of the magnetic field.

\section{Computational model}

The scanning configuration used to study the effect of the TMF on Hanle resonances of $F_{g}=2 \rightarrow F_{e}=3$ transition is shown in Figure 1a.

The direction of propagation of light beam was taken along the $y$ axis and its electric field vector was chosen along $z$ axis. The axis of quantization is defined along the direction of scanning magnetic field ( $z$ axis), which is parallel to the light polarization and perpendicular to the propagation direction of the optical field (transverse scan geometry) (Fig. 1a). This induces $\pi$ transitions (selection rule: $\Delta m=0$ ) between hyperfine levels $F_{g}=2$ and $F_{e}=3$ (Fig. 1b).

The additional TMF, perpendicular to the scanning magnetic field, is applied in the $x y$ plane. The total TMF in the $x y$ plane can be expressed as:

$$
\boldsymbol{B}_{\mathrm{TMF}}=B_{x} \hat{x}+B_{y} \hat{y} .
$$


(a)

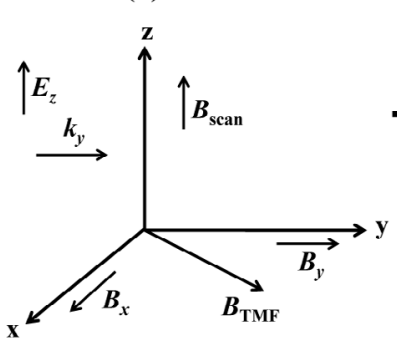

(b)

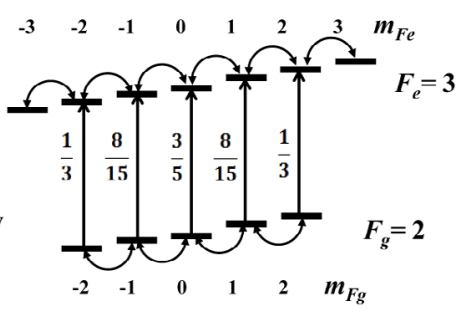

Fig. 1. (a) Scan configuration showing the directions of scanning magnetic field $\left(B_{\text {scan }}\right)$, light wavevector $\left(k_{y}\right)$, light polarization $\left(E_{z}\right)$ and transverse magnetic field $\left(B_{\mathrm{TMF}}\right)$ and $(\mathrm{b})$ atomic level configuration for the transition $F_{g}=2 \rightarrow F_{e}=3$ of ${ }^{87} \mathrm{Rb} D_{2}$ line. The transition probabilities are shown next to the solid arrows [17].

where $B_{x}$ and $B_{y}$ is the magnitude of the TMF along $x$ and $y$ axis, respectively.

The total Hamiltonian $H$ of the system is given by the sum of the unperturbed Hamiltonian $H_{0}$, the atom-light interaction Hamiltonian $H_{I}$, and the atom-magnetic field interaction Hamiltonian $H_{B}$, with

$$
\begin{gathered}
H_{0}=\sum_{i} \hbar \omega_{g_{i}}\left|F_{g}, m_{g_{i}}\right\rangle\left\langle F_{g}, m_{g_{i}}\right| \\
+\sum_{i} \hbar \omega_{e_{i}}\left|F_{e}, m_{e_{i}}\right\rangle\left\langle F_{e}, m_{e_{i}}\right| \\
H_{I}=\sum_{j, k}\left|F_{e}, m_{e_{j}}\right\rangle\left\langle F_{g}, m_{g_{k}}\right|\left[\mathbf{E} \cdot \mathbf{d}_{e_{j} g_{k}}\right]+\text { H.c }
\end{gathered}
$$

where $\mathbf{E}$ is the electric field vector, $\mathbf{d}_{e_{j} g_{k}}=$ $\left\langle F_{e}, m_{e}|e \mathbf{r}| F_{g}, m_{g}\right\rangle$ is the dipole matrix element that couples the sublevels of ground state $\left|F_{g}, m_{g}\right\rangle$ and excited state $\left|F_{e}, m_{e}\right\rangle$, and H.c. is the Hermitian conjugate of the first term in equation (3).

Using the Wigner-Eckart theorem [18,19], the dipole matrix element $\mathbf{d}_{e_{j} g_{k}}$ can be written as:

$$
\begin{aligned}
\mathbf{d}_{e_{j} g_{k}}= & \left\langle F_{e}\|e \mathbf{r}\| F_{g}\right\rangle\left\langle F_{e}, m_{e}|| 1, q ; F_{g}, m_{g}\right\rangle \\
= & \left\langle F_{e}\|e \mathbf{r}\| F_{g}\right\rangle(-1)^{F_{e}+1-m_{g}} \\
& \times\left(\begin{array}{ccc}
F_{e} & 1 & F_{g} \\
-m_{e} & q & m_{g}
\end{array}\right) ; \quad q=0, \pm 1,
\end{aligned}
$$

where the angled brackets denote the reduced matrix element and the term in parenthesis is a $3-j$ symbol.

The total magnetic field-atom interaction Hamiltonian $H_{B}$ is sum of the scanning magnetic field-atom interaction Hamiltonian $H_{\mathrm{B}_{\mathrm{scan}}}$, and the additional TMF-atom interaction Hamiltonian $H_{\mathrm{B}_{\mathrm{TMF}}}$,

$$
H_{\mathrm{B}}=H_{\mathrm{B}_{\text {scan }}}+H_{\mathrm{B}_{\mathrm{TMF}}},
$$

$H_{\mathrm{B}_{\text {scan }}}=g \mu_{B} B_{\text {scan }}$

$\times\left\{\sum_{i} m_{g_{i}}\left|F_{g}, m_{g_{i}}\right\rangle\left\langle F_{g}, m_{g_{i}}\left|+\sum_{i} m_{e_{i}}\right| F_{e}, m_{e_{i}}\right\rangle\left\langle F_{e}, m_{e_{i}}\right|\right\}$,

$$
\begin{aligned}
& H_{\mathrm{B}_{\mathrm{TMF}}}=\sum_{i \neq j} \frac{1}{2} g \mu_{B}\left(B_{x}-i B_{y}\right) \\
& \times\left\{C_{F_{g} m_{g_{i}}}^{+}\left|F_{g}, m_{g_{i}}\right\rangle\left\langle F_{g}, m_{g_{j}}\left|+C_{F_{e} m_{e_{i}}}^{+}\right| F_{e}, m_{e_{i}}\right\rangle\left\langle F_{e}, m_{e_{j}}\right|\right\} \\
& \quad+\sum_{i \neq j} \frac{1}{2} g \mu_{B}\left(B_{x}+i B_{y}\right) \\
& \quad \times\left\{C_{F_{g} m_{g_{i}}}^{-}\left|F_{g}, m_{g_{i}}\right\rangle\left\langle F_{g}, m_{g_{j}}\left|+C_{F_{e} m_{e_{i}}}^{-}\right| F_{e}, m_{e_{i}}\right\rangle\left\langle F_{e}, m_{e_{j}}\right|\right\}
\end{aligned}
$$

where $\mu_{B}$ is the Bohr magneton, and $g$ the gyromagnetic ratio. The Clebsch Gordan coefficient $\left(C_{F_{a} m_{a}}^{ \pm}\right)$, with $a=$ $(e, g)$, is given by:

$$
C_{F_{a} m_{a}}^{ \pm}=\sqrt{\left(F_{a} \mp m_{a}\right)\left(F_{a} \pm m_{a}+1\right)} .
$$

The scanning magnetic field $B_{\text {scan }}$ splits the Zeeman sublevels of $F_{g}=2$ and $F_{e}=3$ (see Fig. 1b) and this splitting is proportional to $B_{\text {scan }}$ (see Eq. (6)).

Since the TMF is perpendicular to the axis of quantization, it couples the Zeeman sublevels of $\Delta m_{F}= \pm 1$ (see Eq. (7)) and causes population redistribution in the ground state $\left(F_{g}=2\right)$ and excited state $\left(F_{e}=3\right)$ sublevels. This is indicated by the curved arrows in Figure 1b.

The time evolution of the density matrix $\rho$ is given by the Liouville equation [20]:

$$
\frac{d \rho}{d t}=\frac{1}{i h}[\bar{H}, \rho]-\frac{1}{2}\{R, \rho\}+\Lambda_{\gamma}+\Lambda_{\Gamma},
$$

where $\bar{H}$ is the total Hamiltonian of the system after making rotating wave approximation. On the right side of equation (8), the first and second terms represent the commutation and anticommutation operations, respectively. $R$ is the diagonal matrix which represents the spontaneous decay rate, $\Gamma$ of the excited state and the ground-state collisional decay rate, $\gamma$. The matrices $\Lambda_{\gamma}$ and $\Lambda_{\Gamma}$ represent the repopulation of the ground state due to the relaxation terms $\gamma$ and $\Gamma$, respectively.

The Bloch equations were obtained after numerically solving equation (8) under steady state conditions $[21,22]$. The Hanle absorption is given by:

$$
\alpha=\sum_{e, g} \frac{2 \sqrt{2} \pi \omega_{F_{e} \rightarrow F_{g}} N}{\hbar c \Omega}\left|\mathbf{d}_{e g}\right|^{2}\left(\operatorname{Im}\left[\rho_{(e, g)}\right]\right)
$$

where $\omega_{F_{e} \rightarrow F_{g}}$ is the frequency difference between the ground and the excited states in the absence of the magnetic field and $N$ is the density of atoms.

\section{Computational results}

The calculated absorption spectra of the pump-probe beam for a closed $F_{g}=2 \rightarrow F_{e}=3$ transition of ${ }^{87} \mathrm{Rb} D_{2}$ line in the absence and presence of the TMF is shown in Figure 2. 


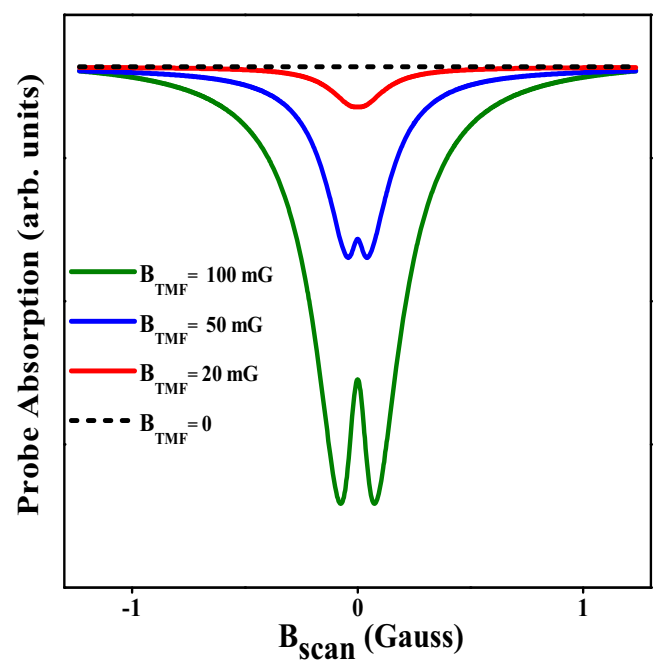

Fig. 2. Numerically computed absorption spectra of $F_{g}=2 \rightarrow$ $F_{e}=3$ transition. The dashed line represents $B_{\mathrm{TMF}}=0$ and the solid line represents $B_{\mathrm{TMF}} \neq 0$. Other parameters used in computation: $\Omega_{R}=40 \mathrm{MHz}, \Gamma=38.1 \mathrm{MHz}$, and $\gamma=10^{-3} \Gamma$.

(a)

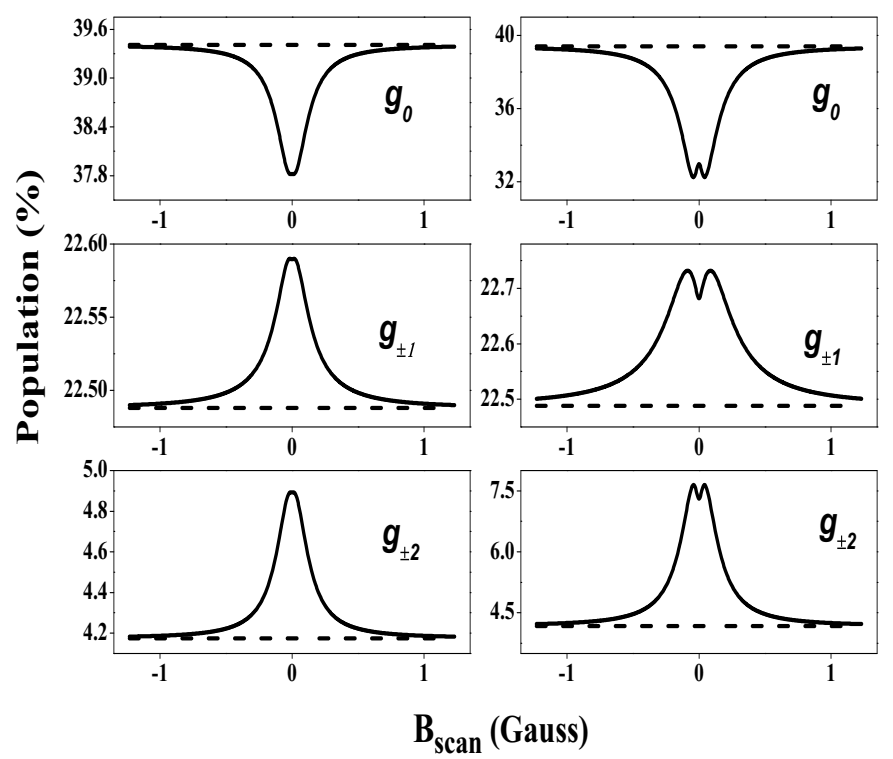

Fig. 3. Population of the Zeeman sublevels of $F_{g}=2$ as function of magnetic field. The dashed lines represent $B_{\mathrm{TMF}}=0$ and the solid lines represent (a) $B_{\mathrm{TMF}}=20 \mathrm{mG}$ and (b) $B_{\mathrm{TMF}}=50 \mathrm{mG}$. Other parameters are same as used in Figure 2.

In the absence of the TMF, no Hanle resonance signal is observed (Fig. 2 (dashed line)). When the TMF is applied, a dip in the absorption signal is observed. With increase in value of the TMF, the absorption signal splits at line centre and two dips are observed on either side of the zero value of the scanning magnetic field (Fig. 2 (solid lines)). These observations can be understood by considering the population distribution among the ground state $\left(F_{g}=2\right)$ sublevels in the absence and presence of the additional TMF (Fig. 3).

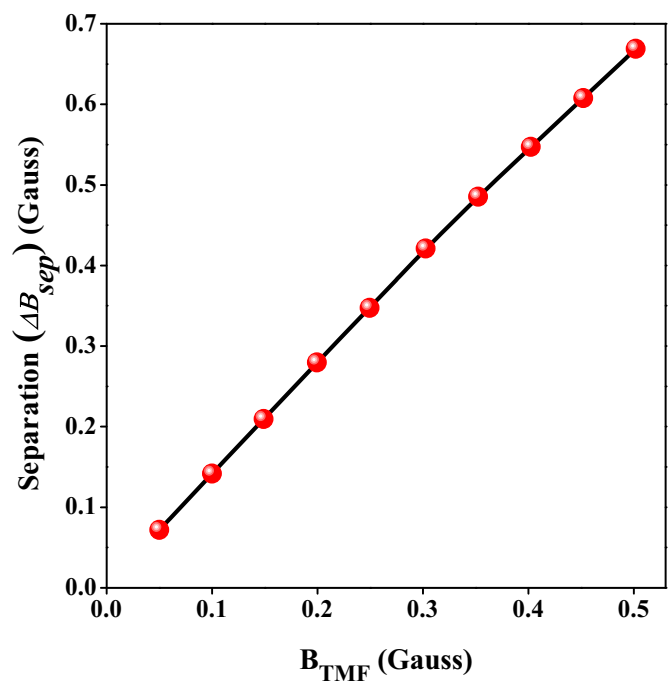

Fig. 4. Numerically calculated separation between the two dips as a function of the TMF. Other parameters are same as used in Figure 2.

The interaction of $\pi$ polarized light with $F_{g}=2 \rightarrow$ $F_{e}=3$ transition results in $\Delta m=0$ transitions among the Zeeman sublevels of ground and excited state. In the absence of the TMF, due to spontaneous decay of the excited state, maximum population of atoms accumulates in the ground state sublevels $g_{0}$ and $g_{ \pm 1}$. But, there is no change of population among ground state sublevels as a function of the scanning magnetic field (Fig. 3 (dashed lines)). As a result no Hanle resonance is obtained (Fig. 2 (dashed line)).

The transition probabilities for selection rule, $\Delta m=0$ ( $\pi$ transition) is shown in Figure 1b. The transition from groundstate sublevel, $g_{0}$ to excited state sublevel, $e_{0}$ has maximum probability, while that from $g_{ \pm 2}$ to $e_{ \pm 2}$ has minimum probability.

At $B_{\text {scan }}=0$, when a small TMF $(=20 \mathrm{mG})$ is applied, the population in $g_{0}$ shifts to $g_{ \pm 1}$ and $g_{ \pm 2}$ sublevels (Fig. 3a (solid lines)), so that the absorption reduces (Fig. 2). As we start scanning the magnetic field, the population of $g_{0}$ starts to increase. Since the transition from $g_{0}$ to excited state sublevel $e_{0}$ has maximum probability, an increase in absorption is observed. When the value of the TMF is increased, two minima are observed in ground state sublevels population (Fig. 3b (solid lines)). These minima correspond to the position of minima in the absorption signal (Fig. 2).

This splitting in the absorption signal is different from the splitting observed with a $\sigma$ polarized light as described in references [23,24]. With $\sigma$ polarized light, absorption minima are observed on either side of the scanning magnetic field, when the population among ground state sublevels become equal. However, no splitting is observed in the ground state population when the magnetic field is scanned. In the present case, absorption minima is observed only when there is a corresponding minima in population (Fig. 3b).

The separation between the two dips is linearly proportional to magnitude of the TMF with a slope $\sim 1.3$ (Fig. 4). 


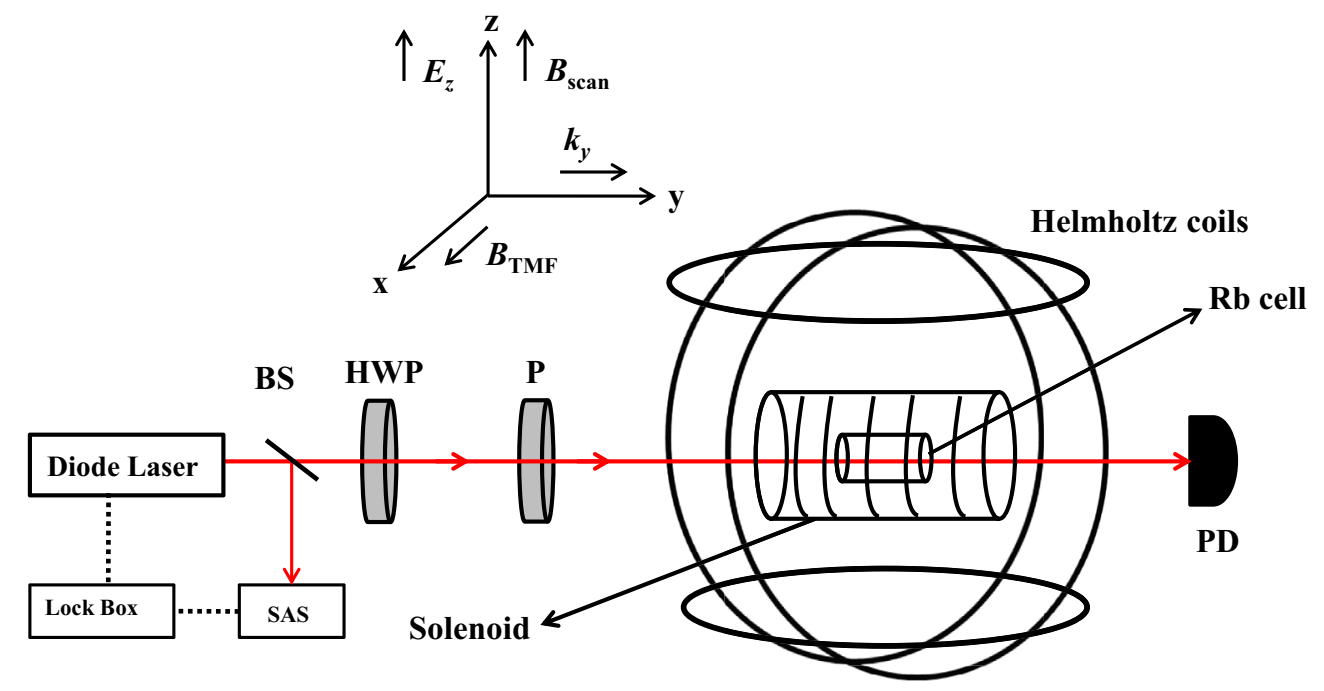

Fig. 5. Schematic of the experimental setup used. Key: BS, beam splitter; HWP, half waveplate; P, polarizer; PD, photo detector and SAS, saturated absorption spectroscopy. A laser beam linearly polarized along the $z$ axis propagates through a $\mathrm{Rb}$ cell. A scanning magnetic field $B_{\text {scan }}$ and a constant magnetic field $B_{\mathrm{TMF}}$ are applied parallel and perpendicular to light polarization direction respectively.

(a)

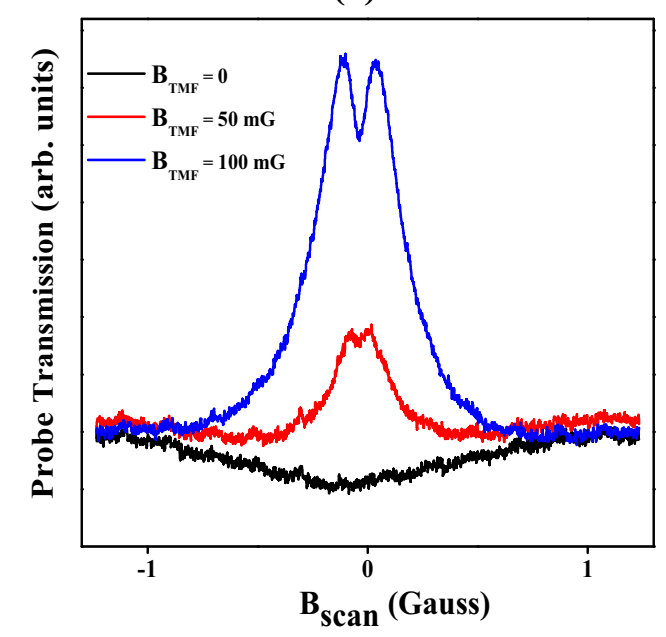

(b)

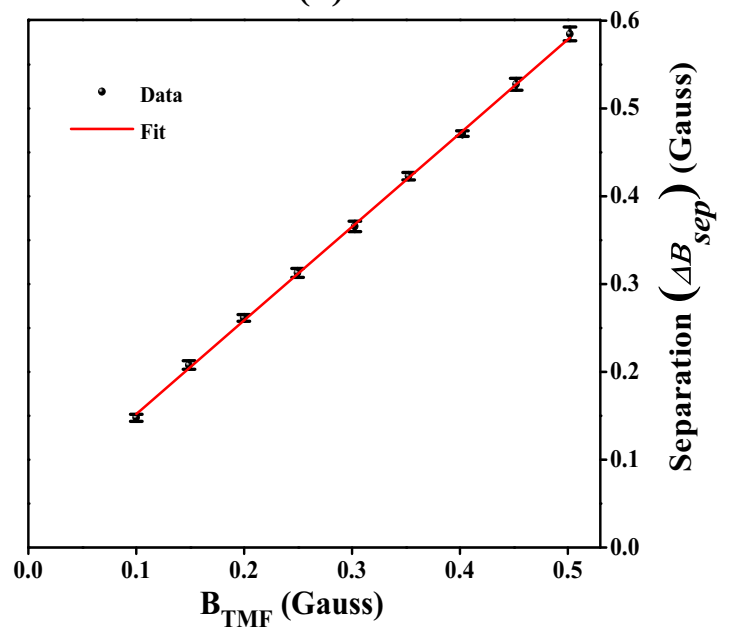

Fig. 6. Experimentally measured (a) laser transmission signal of $F_{g}=2 \rightarrow F_{e}=3$ transition as function of magnetic field, with and without $B_{\mathrm{TMF}}$ and (b) separation between the two peaks as a function of the TMF in the pure Rb cell. The vertical bars show the standard error in the measurement.

We observed similar results for $F_{g}=2 \rightarrow F_{e}=1$, $F_{g}=2 \rightarrow F_{e}=2$ and $F_{g}=1 \rightarrow F_{e}=2$ for both open and closed transitions.

\section{Experimental description}

The experimental setup used to measure resonance signals is shown in Figure 5. A tunable diode laser DL pro (Toptica) was locked to the hyperfine transitions $F_{g}=$ $2 \rightarrow F_{e}=3$ of ${ }^{87} \mathrm{Rb} D_{2}$ line with Toptica Digilock 110. A saturated absorption spectroscopy setup was used to find the exact position of Doppler free hyperfine transitions of ${ }^{87} \mathrm{Rb}$ atoms. The intensity of the laser beam was controlled by using a half wave plate before it passes through $\mathrm{Rb}$ cell in all experiments. A Glan-Thompson polarizer was used to adjust the polarization of a laser beam.

A pure $\mathrm{Rb}$ cell at room temperature $\left(23^{\circ} \mathrm{C}\right)$ is placed at the center of a solenoid and two pairs of coils in Helmholtz configuration, which was used to reduce the Earth's magnetic field to $\sim 0.5 \mathrm{mG}$ along all three axes. The intensity of laser beam was set to $0.18 \mathrm{~mW} / \mathrm{cm}^{2}$ for a pure Rb cell. The laser transmission signal was measured by scanning the magnetic field perpendicular $(z$ axis) to the propagation direction of the laser beam both in the absence and presence of the TMF (Fig. 6a).

The experimental results of Figure $6 \mathrm{a}$ and numerical results of Figure 2 are in good agreement both in the absence and presence of the TMF. 
Figure $6 \mathrm{~b}$ shows the experimentally measured separation between the peaks as function of the TMF. In Figure $6 \mathrm{~b}$, the vertical bars show the standard error in the measurement, and the slope of the curve is $\sim 1.1$.

For a longitudinal scan geometry, scanning magnetic field is parallel to the direction of propagation of the laser beam, " $V$ " type of links can not be formed between Zeeman sublevels of the ground and excited hyperfine levels with a circularly polarized light $\left(\sigma^{+}\right.$or $\left.\sigma^{-}\right)$. Therefore, no Hanle resonance was observed for $F_{g}=2 \rightarrow F_{e}=3$ transition in the absence of the TMF [25]. Similar conditions (no " $V$ " type links) can be obtained with a $\pi$ polarized light in the transverse scan geometry (Fig. 1b), scanning magnetic field is perpendicular to the direction of propagation of the laser beam. Like a circularly polarized light, there is no Hanle resonance observed with a $\pi$ polarized light in the absence of the TMF (Fig. 6a).

With $\pi$ polarized light, a splitting is observed in the transmission signal in the presence of TMF. The separation between the two peaks is linearly proportional to the magnitude of the TMF (Fig. 6). On the other hand, with a circularly polarized light, no splitting is observed when the TMF is introduced [25].

The effect of TMF on Hanle resonances for $F_{g}=2 \rightarrow$ $F_{e}=3$ transition was also studied with a Rb cell containing 30 Torr Ne buffer gas. The Earth's magnetic field was compensated by using a four layer $\mu$-metal shield up to $\sim 0.03 \mathrm{mG}$.

Helmholtz coils to further reduce the residual magnetic field and to scan the magnetic field perpendicular to the propagation direction of the laser beam were installed in the $\mu$-metal shield. The buffer gas cell was heated to a temperature of $70{ }^{\circ} \mathrm{C}$ by passing hot water around the cell holder. The intensity of the laser beam was set to $2.7 \mathrm{~mW} / \mathrm{cm}^{2}$. The measured separation between the two peaks in the transmission signal of a Rb buffer gas cell is linearly proportional to the TMF with a slope $\sim 1.0$ (Fig. 7) which is similar to the results observed for the pure $\mathrm{Rb}$ cell.

Since the separation between the two peaks is linearly proportional to the magnitude of TMF, it can be used for in-situ magnetic field measurements. The sensitivity of this technique is determined by the smallest detectable change in separation between two peaks. With a pure $\mathrm{Rb}$ cell, the measurements were performed within the range of 0.1 to $0.5 \mathrm{G}$ and the magnetic field was increased by $0.05 \mathrm{G}$ in each step (Fig. 6b).

The buffer gas cell allows us to observe the splitting for TMF values in the milligauss region. With a $\mathrm{Rb}$ buffer gas cell, we applied this technique in the range from 10 to $30 \mathrm{mG}$ and minimal change in magnetic field was $1 \mathrm{mG}$ (Fig. 7). We applied this technique to detect only static magnetic fields. A single measurement was performed in $25 \mathrm{~ms}$ time by sweeping the perpendicular magnetic field, and then it was averaged 10 times to improve signal-tonoise ratio.

Some advantages of the current method are, that only a single optical field is used, without the need for any amplitude or frequency modulation. Also, the field can

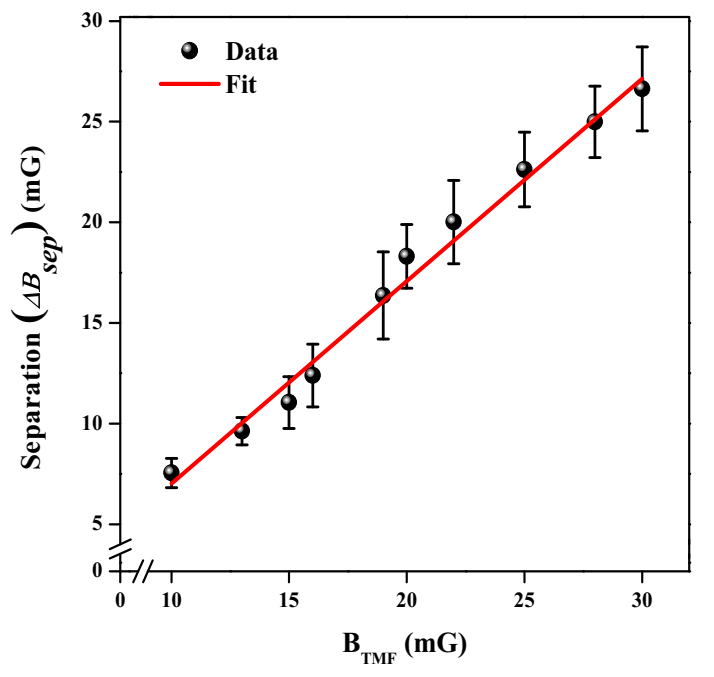

Fig. 7. Experimentally measured separation between the two peaks as a function of the TMF in the Rb cell contains 30 Torr of Ne as buffer gas. The vertical bars show the standard error in the measurement. The breaks $(/ /)$ on the axes indicate the adjustment in the scale.

be directly calculated from the Hanle transmission signal via software processing, without any additional electronic devices.

\section{Conclusion}

We have described a new technique to measure static magnetic fields using Hanle resonances. The magnetic field to be measured (TMF) creates two peaks in the Hanle transmission signal. The separation between the peaks is linearly proportional to the magnitude of the TMF. Therefore, this phenomenon can be implemented in magnetometry. In the present study we have used this technique to measure fields as low as $0.1 \mathrm{G}$ with a pure $\mathrm{Rb}$ cell and as low as $10 \mathrm{mG}$ with a $\mathrm{Rb}$ cell contains 30 Torr of $\mathrm{Ne}$ as buffer gas. It would be possible to measure lower magnetic fields with higher buffer gas pressure and paraffin coated cells.

This work was supported by the Department of Science and Technology, India.

\section{References}

1. D. Budker, D.F. Kimball, S.M. Rochester, V.V. Yashchuk, M. Zolotorev, Phys. Rev. A 62, 043403 (2000)

2. D. Budker, W. Gawlik, D.F. Kimball, S.M. Rochester, V.V. Yashchuk, A. Weis, Rev. Mod. Phys. 74, 1153 (2002)

3. D. Budker, D.F. Kimball, V.V. Yashchuk, M. Zolotorev, Phys. Rev. A 65, 055403 (2002)

4. W. Gawlik, L. Krzemie, S. Pustelny, D. Sangla, J. Zachorowski, M. Graf, A.O. Sushkov, D. Budker, Appl. Phys. Lett. 88, 131108 (2006)

5. W.E. Bell A.L. Bloom, Phys. Rev. 107, 1559 (1957) 
6. J. Dupont-Roc, S. Haroche, C. Cohen-Tannoudji, Phys Lett. A 28A, 638 (1969)

7. C. Cohen-Tannoudji, J. DuPont-Roc, S. Haroche, F. Laloë, Phys. Rev. Lett. 22, 758 (1969)

8. A. Nagel, L. Graf, A. Naumov, E. Mariotti, V. Biancalana, D. Meschede, R. Wynands, Europhys. Lett. 44, 31 (1998)

9. M. Stahler, S. Knappe, C. Affolderbach, W. Kemp, R. Wynands, Europhys. Lett. 54, 323 (2001)

10. L. Guobin, G. Sihong, J. Phys. B 43, 035004 (2010)

11. D. Budker, M.V. Romalis, Nat. Phys. 3, 227 (2007)

12. F. Renzoni, W. Maichen, L. Windholz, E. Arimondo, Phys. Rev. A 55, 3710 (1997)

13. Y. Dancheva, G. Alzetta, S. Cartaleva, M. Taslakov, C. Andreeva, Opt. Commun. 178, 103 (2000)

14. F. Renzoni, S. Cartaleva, G. Alzetta, E. Arimondo, Phys. Rev. A 63, 065401 (2001)

15. N. Ram, M. Pattabiraman, C. Vijayan, Phys. Rev. A 82 033417 (2010)

16. G. Raghwinder, M. Pattabiraman, J. Phys. B 48, 085501 (2015)

17. D.A. Steck, Rubidium 87 d line data, http://steck.us/ alkalidata (revision 2.1.4, 23 December 2010)

18. D.M. Brink, G.R. Satchler, Angular Momentum (Oxford, 1962)
19. J.L. Meunier, Eur. Phys. J. 8, 114 (1987)

20. M.O. Scully, M.S. Zubairy, Quantum Optics (Cambridge University Press, 1997)

21. S. Rochester, Computed with a user-extensible mathematica based atomic density matrix package, http://budker. berkeley.edu/ADM (2012), a detailed account of the theoretical framework can be found at reference [22]

22. Yu. P. Malakyan, S.M. Rochester, D. Budker, D.F. Kimball, V.F. Yashchuk, Phys. Rev. A 69, 013817 (2004)

23. L. Margalit, M. Rosenbluh, A.D. Wilson-Gordon, Phys. Rev. A 87, 033808 (2013)

24. G. Raghwinder, M. Pattabiraman, J. Phys. B 47, 195501 (2014)

25. J. Dimitrijević, A. Krmpot, M. Mijailović, D. Arsenović, B. Panić, Z. Grujić, B.M. Jelenković, Phys. Rev. A 77, 013814 (2008)

Open Access This is an open access article distributed under the terms of the Creative Commons Attribution License (http://creativecommons.org/licenses/by/4.0), which permits unrestricted use, distribution, and reproduction in any medium, provided the original work is properly cited. 